\title{
Role of anticipatory responses in avoidance conditioning: An important control'
}

\author{
M. R. D'AMATO \\ RUTGERS UNIVERSITY
}

Substantial numbers of "avoidance" (anticipatory) responses are maintained in avoidance conditioning paradigms though no avoidance contingency is operating. This suggests that variables other than the avoidance contingency are responsible for the emergence of $\mathrm{CRs}$, and that variables which influence avoidance acquisition might do so because of their influence on anticipatory responding. It is suggested that the effect of such variables on antic ipatory responding be routinely assessed by including control groups in which the avoidance contingency is witheld.

The purpose of this paper is to call attention to the vital role played by "anticipatory" responses in avoidance conditioning and to point out the desirability of assessing their contribution by appropriate control groups. By anticipatory responses I refer to instrumental responses which occur during the CS-US interval in the absence of an avoidance contingency. In classical aversive conditioning such responses provide the definition and measure of conditioning. However, it is not generally recognized that anticipatory responding plays an exceedingly important role in avoidance conditioning. Because an avoidance contingency is programmed in such paradigms, it is natural to attribute the observed CRs to the contingency itself.

Many years ago Mowrer \& Lamoreaux (1942) pointed out the important role played by anticipatory responses in avoidance conditioning. Except for the devising of techniques to "correct" avoidance scores for "operant levels," their observation went largely unheeded. My own attention was directed to the importance of anticipatory responses by the following results. Method

As part of a larger experiment investigating variables influencing discriminated avoidance conditioning in the lever-press situation (D'Amato, Fazzaro, \& Etkin, 1967), we ran two groups of 14 albino rats in which the avoidance contingency was eliminated. In Group A there was prompt CS and US (discontinuous shock) termination on escape trials. On CR trials (i.e., trials on which $\mathrm{S}$ made an anticipatory response) nothing at all happened, and the US was delivered at the end of the 5-sec CS-US interval; S had to respond again in order to escape shock. Group B differed from Group A only in that prompt CS termination was never associated with the escape response; rather the CS persisted for $8 \mathrm{sec}$ beyond execution of the latter. These groups are similar to what Kamin (1956) has referred to as "classical" groups.

Resulis and Discussion

Figure 1 shows the results obtained from Groups A and $B$ over 300 acquisition trials given in a single day. For comparison purposes the results of a control group (Group C) for which the avoidance contingency was in effect are included. It is evident from the figure that a substantial percentage of CRs is maintained in Groups $A$ and B, though of course, the controls responded at a higher level. The difference between Groups $A$ and $B$ is not significant $(t=1.36)$. We had occasion to replicate the conditions of Group B with eight Ss and found an even greater level of anticipatory responding, approximately 30 percent CRs over the 300 trials.

In other experiments in which "classical" groups have been run, substantial amounts of anticipatory responding have also been observed. Kamin's classical group reached a level of better than 30 percent CRs before tapering off. In the experiments reported by Bolles, Stokes, \& Younger (1966), both a shuttle box and a running wheel were employed, and substantial numbers of CRs were observed in the groups not having the benefit of an avoidance contingency. It is clear, then, that in a wide range of apparatuses and experimental situations CRs may occur to a very significant extent even though an avoidance contingency is specifically excluded.

These results suggest that where an avoidance contingency is in effect, initial CRs are generated by factors other than the avoidance contingency itself. I do not wish to go into detail here as to what these other factors might be, but clearly termination of the US by the instrumental response is a potent agent, and as our results suggest, prompt CS termi-

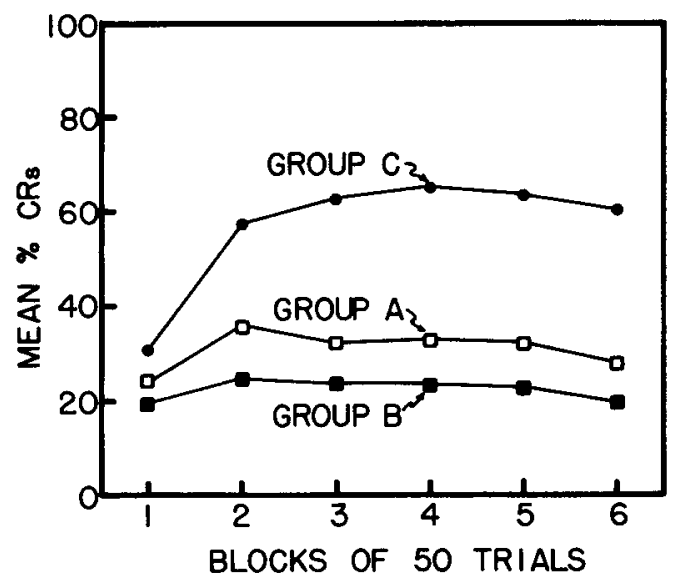

Fig. 1. Percent CRs over 300 acquisition trials. There was no avoidance contingency operating in Groups $A$ and $B$. 
nation by the instrumental response may also contribute toward this end. If the instrumental response arises as an "unconditioned response" to the US, as the response of running in a wheel to shock, then responseproduced US termination is apparently not necessary for the generation of substantial numbers of anticipatory responses (e.g., Bolles et al, 1966). With more involved responses, such as bar pressing, response-produced termination of the US is probably necessary, though US termination need not be immediate.

In any event, it seems plain that any variable which acts to affect importantly the level of anticipatory responding obtained is bound to have a commensurate effect on avoidance conditioning. To cite one illustration, it is well documented that relatively strong shock interferes with avoidance acquisition in the bar-press situation (e.g., D'Amato \& Fazzaro, 1966). It seems likely that the basis of this result is that strong shock reduces anticipatory responding, a hypothesis which can be easily evaluated by manipulating shock intensity while withholding the avoidance contingency. 2 Other variables which have been shown to influence avoidance conditioning might operate in a similar way, for example, type of US (discontinuous shock, D'Amato, Keller, \& DiCara, 1964), type of apparatus (Theios, Lynch, \& Lowe, 1966), and certain physiological manipulations (e.g., Trafton, 1967).

As soon as the avoidance contingency is eliminated the problem arises that on CR trials the instrumental response suffers some degree of punishment. In our situation this proved not to be a very significant factor, inasmuch as the probability of a CR after a CR was just about the same as after an escape response. Possibly this was because the CRs were often of low latency and hence a few seconds separated the $\mathrm{CR}$ and delivery of the US. Where punishment does suppress anticipatory responding, one can at least be certain that such responses must occur to an even greater extent in the groups for which the avoidance contingency is in operation. Possibly some amelioration of the effects of punishment can be obtained by postponing the application of the US after a CR for some minimal interval, perhaps 2 or $3 \mathrm{sec}$.

In conclusion, it is urged that experimenters in- vestigating variables that influence avoidance conditioning include appropriate control groups to assess the effect of their independent variables on anticipatory responses. If a given independent variable facilitates avoidance conditioning while at the same time raising substantially anticipatory responding, it is reasonable to conclude that at least some of its effect on the former is through the latter mechanism. Conversely, if such a variable had no effect on anticipatory responding, this would indicate that it exerted its influence elsewhere, as we have suggested perhaps on discrimination of the avoidance contingency (D'Amato et al, 1967). Another function which such control groups might serve is to provide a somewhat better index of the level of CRs to be expected in the absence of the avoidance contingency.

\section{References}

Bolles, R. C., Stokes, L. W., \& Younger, M. S. Does CS termination reinforce avoidance behavior? J. comp. physiol. Psychol., 1966, 62, 201-207.

D'Amato, M. R., \& Fazzaro, J. Discriminated lever-press avoidance learning as a function of type and intensity of shock. J. comp. physiol. Psychol., 1966, 61, 313-315.

D'Amato, M. R., Fazzaro, J., \& Etkin, M. Anticipatory responding and avoidance discrimination as factors in avoidance conditioning. Submitted to J. exp. Psychol., April, 1967.

D'Amato, M. R., Keller, D., \& DiCara, L. Facilitation of discriminated avoidance learning by discontinuous shock. J. comp. physiol. Psychol., 1964, 58, 344-349.

Kamin, L. J. The effects of termination of the CS and avoidance of the US on avoidance learning. J. comp. physiol. Psychol., $1956,49,420-424$

Mowrer, O. H., \& Lamoreaux, R. R. Avoidance conditioning and signal duration-a study of secondary motivation and reward. Psychol. Monogr., 1942, 54, No. 5 (Whole No. 247).

Theios, J., Lynch, A. D., \& Lowe, W. F., Jr. Differential effects of shock intensity on one-way and shuttle avoidance conditioning. J. exp. Psychol., 1966, 72, 294-299.

Trafton, C. L. Effects of lesions in the septal area and cingulate cortical areas on conditioned suppression of activity and avoidance behavior in rats. $J$. comp. physiol. Psychol., 1967,63, 191-197.

\section{Notes}

1. This research was supported by National Science Foundation grant GB-4011.

2. An interpretation which squares with the interesting fact that shock level is positively related to avoidance maintenance (D'Amato, M. R., Fazzaro, J., \& Etkin, M. Discriminated barpress avoidance maintenance and extinction in rats as a function of shock intensity. J. comp. physiol. Psychol., 1967, 63, 351-354). 